\title{
Research on RSA-based Broadcast Encryption Scheme in Web Multimedia
}

\author{
Liang Xue \\ College of Computer Science \\ Sichuan University \\ Chengdu, China, 13541243587 \\ liangxue_scu@163.com
}

\author{
Du Zhongjun \\ College of Computer Science \\ Sichuan University \\ Chengdu, Sichuan, 13618066736 \\ duzhongjun@scu.edu.cn
}

\begin{abstract}
With the development of web multimedia technology, broadcast encryption schemes play an important role in multimedia services copyright protection. In this paper, we propose a new broadcast encryption scheme based on RSA, besides, we give out two algorithms-smallest power exponent algorithm and improved modular exponential algorithm, to simplify the repeated squaring in RSA. This scheme could reduce the encryption and decryption calculated amount greatly, improve the communication bandwidth and deter the forcible attack effectively. In the course of KGC(Key Generation Center) distributing keys, we adopt the different module remainder strategy, thus realizing that when new members jion in, keys needn't be updated, and this could enhance the practicality and security of broadcast encryption scheme.
\end{abstract}

Keywords-web multimedia; RSA; broadcast encryption

\section{INTRODUCTION}

Web multimedia, the development of traditional multimedia services on web, is one of the hotspot of computer application technique researches now. With the development of web multimedia technology, broadcast encryption schemes play an important role in multimedia services copyright protection. The broadcast encryption[1], which provides a safe way to distribute digital information for authorized users over the broadcast channel, has a broad application prospect in the pay$\mathrm{TV}$, video conference and other occasions. In this paper, we propose a new broadcast encryption scheme based on RSA, besides, we give out two algorithms - smallest power exponent algorithm and improved modular exponential algorithm, to simplify the repeated squaring in RSA. Our scheme could reduce the encryption and decryption calculated amount greatly, improve the communication bandwidth and deter the forcible attack effectively. Moreover, in the course of distributing keys, we adopt the different module remainder strategy, thus realizing that when new members jion in, keys needn't be updated, in addition, the storage amount of keys is greatly increased.

\section{RELATED WORK}

The notion of broadcast encryption was first put forward by Berkovits[2] in 1991. Later, Fiat and Noar[3] gave a formal study of broadcast encryption and proposed a mechanism to prevent collusion. Since then, Broadcast encryption received extensive attention, many broadcast encryption schemes were proposed to enhance the development of this field. Naor and Pinkas[4] performed a public key broadcast encryption scheme which used a threshold secret sharing method. Tan Zuowen et al.[5] introduced a fully public key tracing and revocation scheme, and the salient feature of the scheme was that the secret keys of the users were chosen by the users themselves. Li Xiaofeng et al.[6] according to the protocol of RSA and the enlarging the small public key technology presented a new broadcast encryption scheme of RSA oriented multi-recipient. At the same time, in order to simplify the key management, some scholars put forward the identity based broadcast encryption scheme, for example, Sun Jin et al.[7] performed a novel identity-based broadcast encryption scheme by combining with Waters dual system encryption and the orthogonality property of composite-order bilinear groups.

\section{RSA-BASED BROADCAST ENCRYPTION SCHEME}

In our scheme, there are three entities:

Data Provider(DP): A data provider in our scheme is responsible for providing and encrypting multimedia content.

Key Generation Center(KGC): In this scheme, it is in charge of achieving system initialization, key generation, key distribution and user management.

Authorized User(AU): An authorized user is a subscriber who could receive the multimedia content.

\section{A. Initialization}

We assume that all the data transmit through security authentication channel. The procedure works as follows.

DP chooses two distinct prime numbers $\mathrm{p}$ and $\mathrm{q}$ randomly, computes $\mathrm{n}=\mathrm{pq}$ and $\varphi(\mathrm{n})=(\mathrm{p}-1)(\mathrm{q}-1)$, where $\varphi$ is Euler's totient function, and chooses an integer e such that $1<\mathrm{e}<\varphi(\mathrm{n})$ and greatest common divisor of $(\mathrm{e}, \varphi(\mathrm{n}))=1$. So the public key for DP is $(e, n)$.

For all authorized users $\mathrm{au}_{\mathrm{i}}, \mathrm{i}=1,2,3, \ldots \ldots, \mathrm{s}(\mathrm{s}$ is the number of AUs), they all send their own identity information $\mathrm{ID}_{\mathrm{i}}$ to $\mathrm{KGC}$, and $\mathrm{KGC}$ adds $\left(\mathrm{ID}_{\mathrm{i}}, \mathrm{d}_{\mathrm{i}}, \mathrm{R}_{\mathrm{i}}\right)$ to the storage list, where $d_{i} \cdot e \equiv 1 \bmod \left(R_{i} \cdot \varphi(n)\right)$, and $R_{i}$ is a random prime number.

In the end, KGC sends the private key $\left(\mathrm{d}_{\mathrm{i}}, \mathrm{n}\right)$ to aui safely and accurately. 


\section{B. Encryption}

In the light of RSA algorithm, we assume that the plaintext $\mathrm{m}=\left(\mathrm{m}_{1}, \mathrm{~m}_{2}, \mathrm{~m}_{3}, \ldots \ldots, \mathrm{m}_{\mathrm{k}}\right)$ and the ciphertext $\mathrm{c}=\left(\mathrm{c}_{1}, \mathrm{c}_{2}\right.$, $\left.c_{3}, \ldots \ldots, c_{k}\right)$, so the encryption algorithm $E$ is thus:

$$
\mathrm{c}_{\mathrm{j}}=\mathrm{m}_{\mathrm{j}}{ }^{\mathrm{e}}(\bmod \mathrm{n}), \mathrm{j}=1,2,3, \ldots \ldots, \mathrm{k} .
$$

Note that the encryption procedure need $\mathrm{k}$ times modular exponential operations, obviously, the encryption speed is slow and need a significant amount of bandwidth.

While in our paper, we propose a new encryption algorithm, where the process is:

$$
c_{j}=\left(m_{j-1+} m_{j}\right)(\bmod n), i=k, k-1, \ldots \ldots, 3,2
$$

and

$$
\mathrm{c}_{1}=\mathrm{m}_{1}{ }^{\mathrm{e}}(\bmod \mathrm{n}) .
$$

So we only use one time modular exponential operation and k-1 times modular add operations, which considerably reduces the time of encryption.

Moreover, for the modular exponential operation, we convert exponent $\mathrm{m}$ to binary number for repeated squaring operation traditionally. Howerver, in practical applications, exponent $\mathrm{m}$ is required to be large to enhance security, so the computation speed is still slow for encryption procedure. In our paper, we give out two algorithms - smallest power exponent algorithm and improved modular exponential algorithm, to simplify the repeated squaring in RSA. This scheme could reduce the encryption and decryption calculated amount effectively and greatly improve the communication bandwidth.

\section{1. smallest power exponent algorithm}

Judge whether $\mathrm{n}$ is a prime in the formula $\mathrm{m}^{\mathrm{e}}(\bmod \mathrm{n})$ firstly.

Then if it is, compute $\varphi(n)$ and $r$, where $r$ is remainder of deviding $\varphi(n)$ by e, thus $r$ is final smallest power exponent.

Then if not, factorize integer $n=p_{1}{ }^{v_{1}} p_{2}{ }^{v_{2}} p_{3}{ }^{v_{3}} \ldots . . . p_{k}{ }^{v_{k}}$, and figure out $\varphi\left(\mathrm{p}_{1}^{\mathrm{v}_{1}}\right), \varphi\left(\mathrm{p}_{2}{ }^{\mathrm{v}_{2}}\right), \varphi\left(\mathrm{p}_{3}{ }^{\mathrm{v}_{3}}\right), \ldots \ldots, \varphi\left(\mathrm{p}_{\mathrm{k}}{ }^{\mathrm{v}_{\mathrm{k}}}\right)$ respectively, let $\lambda=\left[\varphi\left(p_{1}^{v_{1}}\right), \varphi\left(p_{2}{ }^{v_{2}}\right), \varphi\left(p_{3}{ }^{v_{3}}\right), \ldots \ldots, \varphi\left(p_{k}{ }^{v_{k}}\right)\right]$, and compute $\mathrm{e} \equiv \mathrm{r}(\bmod \lambda)$, thus $\mathrm{r}$ is final smallest power exponent.

\section{2. improved modular exponential algorithm}

First, we let $\mathrm{m}_{0}=\mathrm{m}$ and $\mathrm{e}_{0}=\mathrm{e}$, compute $\mathrm{m}_{0}{ }^{2}, \mathrm{~m}_{0}{ }^{3}, \ldots \ldots$, until finding out a $\mathrm{q}_{0}$, satisfying $\mathrm{m}_{0}{ }^{\mathrm{q}_{0}}>\mathrm{n}$ and $\mathrm{m}_{0}{ }^{\mathrm{q}_{0}-1}<\mathrm{n}$. If no $\mathrm{q}_{0}$ meet this condition, then compute $\mathrm{m}^{\mathrm{e}} \equiv \mathrm{x}(\bmod \mathrm{n})$ directly; if having an appropriate $\mathrm{q}_{0}$, compute $\mathrm{m}_{0}{ }^{\mathrm{q}_{0}} \equiv \mathrm{m}_{1}(\bmod \mathrm{n})$, $\mathrm{e}_{0}=\mathrm{q}_{0} \mathrm{e}_{1}+\mathrm{r}_{1}$, where $1 \leq \mathrm{r}_{1}<\mathrm{q}_{0} \leq \mathrm{e}_{0}, \quad \mathrm{~m}_{0}{ }^{\mathrm{r}_{1}}=\mathrm{b}_{1}$, so we could transform $\mathrm{m}^{\mathrm{e}}(\bmod \mathrm{n})$ to $\mathrm{m}_{1}{ }^{\mathrm{e}_{1}} \mathrm{~b}_{1}(\bmod \mathrm{n})$;

Similarly, make a calculation of $\mathrm{m}_{1}{ }^{2}, \mathrm{~m}_{1}{ }^{3}, \ldots \ldots$, until finding out a $\mathrm{q}_{1}$, satisfying $\mathrm{m}_{1} \mathrm{q}_{1}>\mathrm{n}$, and $\mathrm{m}_{1} \mathrm{q}_{1}-1<\mathrm{n}$. If no $\mathrm{q}_{1}$ meet this condition, then compute $\mathrm{m}_{1}{ }^{\mathrm{e}_{1}} \mathrm{~b}_{1} \equiv \mathrm{x}_{1}(\bmod \mathrm{n})$ directly; if having an appropriate $\mathrm{q}_{1}$, compute $\mathrm{m}_{1}{ }^{\mathrm{q}_{1}} \equiv \mathrm{m}_{2}(\bmod \mathrm{n})$, $\mathrm{e}_{1}=\mathrm{q}_{1} \mathrm{e}_{2}+\mathrm{r}_{2}$, where $1 \leq \mathrm{r}_{2}<\mathrm{q}_{1} \leq \mathrm{e}_{1}, \quad \mathrm{~m}_{1}^{\mathrm{r}_{2}}=\mathrm{b}_{2}$, so we could transform $\mathrm{m}^{\mathrm{e}}(\bmod \mathrm{n})$ to $\mathrm{m}_{2}{ }^{\mathrm{e}_{2}} \mathrm{~b}_{2} \mathrm{~b}_{1}(\bmod \mathrm{n})$;

In the encryption process, e is a definite number, so by the finite-step calculation, $\mathrm{m}^{\mathrm{e}}(\bmod \mathrm{n})$ is transformed to the following formula:

$$
\mathrm{m}_{\mathrm{k}}{ }^{\mathrm{e}_{\mathrm{k}}} \mathrm{b}_{\mathrm{k}} \mathrm{b}_{\mathrm{k}-1} \ldots \mathrm{b}_{1}(\bmod \mathrm{n}) .
$$

As can be seen from above, we could get the final answer only by $\mathrm{k}$ times multiplication and $\bmod \mathrm{n}$ operations. This could reduce the calculation amount of modular exponential operation effectively.

\section{Decryption}

Assume that the plaintext $\mathrm{m}=\left(\mathrm{m}_{1}, \mathrm{~m}_{2}, \mathrm{~m}_{3}, \ldots \ldots, \mathrm{m}_{\mathrm{k}}\right)$, correspondingly, the ciphertext $\mathrm{c}=\left(\mathrm{c}_{1}, \mathrm{c}_{2}, \mathrm{c}_{3}, \ldots \ldots, \mathrm{c}_{\mathrm{k}}\right)$, so the decryption algorithm for traditional RSA is thus:

$$
m_{j}=c_{j}^{d_{i}}(\bmod n), j=1,2,3, \ldots \ldots, k .
$$

Similarly, in our scheme, we use a new RSA decryption algorithm:

$$
\mathrm{m}_{1}=\mathrm{c}_{\mathrm{j}} \mathrm{d}_{\mathrm{i}}(\bmod \mathrm{n})
$$

and

$$
m_{j}=\left(c_{j}-m_{j-1}\right)(\bmod n), j=2,3,4, \ldots \ldots k-1, k .
$$

So we only use one time modular exponential operation and k-1 times modular add operations, which considerably reduces the time of decryption.

Besides, we also use smallest power exponent algorithm and improved modular exponential algorithm to reduce the decryption calculation.

\section{SECURITY ANALYSIS}

In the RSA-based broadcast encryption scheme, public key $(e, n)$ is open to all, i.e., eavesdroppers could get the value of $\mathrm{e}$ and $\mathrm{n}$. If a eavesdropper attempts to crack the ciphertext, he need know the private key $(d, n)$, where $d \equiv e^{-1}(\bmod \varphi(n))$, e and $\mathrm{n}$ are known. The crucial point to get $\mathrm{d}$ is from the value of $\mathrm{p}^{*} \mathrm{q}$ to get the value of (p-1) and (q-1), but it is a recognized mathematical problem to divide the product of two large prime numbers, moreover, when the product of $\mathrm{p}$ and $\mathrm{q}$ is as large as 1024 bits, it is out of the question to complete the factorization so far. For this reason, RSA is considered to be one of the best public key algorithms. In our paper, we apply RSA algorithm to our broadcast encryption scheme, unauthorized users will not get the private key $\left(\mathrm{d}_{\mathrm{i}}, \mathrm{n}\right)$ the $\mathrm{KGC}$ sends to $\mathrm{AU}$, and they could not get it by collusion.

In RSA applications, $p$ and $q$ must be large enough prime numbers to ensure the safety of RSA and avoid being forcibly attacked, only in this way, can we make attackers not divide the product of two large prime numbers in polynomial time. However, as the bit of private key increases, the timeconsuming modulo exponentiation computation in encryption and decryption procedures, which has always been the bottle- 
neck of RSA, restricts its wider development. In our paper, the new scheme significantly lowers the computational complexity, and greatly reduces the burden of larges keys in RSA, thus we could deter the forcible attack effectively.

\section{CONCLUSION}

We propose a new RSA-based broadcast encryption in web multimedia. In the course of KGC distributing keys, we adopt the different module remainder strategy, thus realizing that when new members jion in, keys needn't be updated, and this could enhance the practicality and security of broadcast encryption scheme. In the process of encryption and decryption, we use one time modular exponential operation and k-1 times modular add operations instead of traditional $\mathrm{k}$ times modular exponential operations to reduce the time of encryption and decryption. Besides, we use two new algorithms - smallest power exponent algorithm and improved modular exponential algorithm to simplify the repeated squaring in RSA. With the acceleration of computation, the scheme could greatly reduces the burden of larges keys in RSA, thus we could deter the forcible attack effectively.

\section{REFERENCES}

[1] Chen Yanli, Yang Geng and Cao Xiaomei. "A survey of research on broadcast encryption," Computer Technology and Development, 2010, Vol. 20, pp. 189-193

[2] S. Berkovits, "How to broadcast a secret," Proceedings of the 10th Annual International Conference on Theory and Application of Cryptographic Techniques, 1991, pp. 536-541.

[3] A. Fiat and M. Naor, "Broadcast encryption," Proceedings of the 13th Annual International Cryptology Conference on Advances in Cryptology, 1993, pp. 480-491.

[4] M. Naor and B. Pinkas, "Efficient trace and revoke schemes," Proceedings of the 4th International Conference on Financial Cryptography, 2001, pp. 1-20.

[5] Tan Zuowen, Liu Zhuojun and Xiao Hongguang, "A fully public key tracing and revocation scheme provaly secure against adptive adversary," Joural of Software, 2005, Vol. 16, pp. 1333-1343

[6] Li Xiaofeng, Lu Jianzhu and Wang Meng, "A new broadcast encryption scheme based on RSA," Microcomputer Information, 2006, Vol. 22, pp. 59-60

[7] Sun Jin and $\mathrm{Hu}$ Yupu, "Identity-based broadcast encryption scheme using the new techniques for dual system encryption," Journal of Electronics \& Information Technology, 2011, Vol. 33, pp. 1266-1270 